\title{
Perfil epidemiológico de gestantes/puérperas soropositivas para o HIV em uma maternidade de referência em Belém-PA
}

\author{
Epidemiological profile of HIV-positive pregnant women / postpartum women in a \\ reference maternity hospital in Belém-PA
}
Perfil epidemiológico de mujeres embarazadas con $\mathrm{VIH} /$ mujeres posparto en un hospital de maternidad de referencia en Belém-PA

Adilson Mendes de Figueiredo Júnior ${ }^{1 *}$, Silvia Cristina Santos da Silva ${ }^{1}$, Glória Letícia Oliveira Gonçalves Lima ${ }^{1}$, Brena dos Santos Pimenta', Elizangela Fonseca de Mendonça', Élen Gabriela Sales Costa', Gabriel Maia Franco', Fernanda Furtado da Cunha², Braydson Nunes dos Santos², Luciana Nascimento Duarte Rodrigues², John Lennon Santos Martins's, Isys Penedo de Matos², Mayara Melo Galvão², Ana Rosa Tavares da Paixão³, Barbara Alves Ruela de Azevedo3.

\section{RESUMO}

Objetivo: Descrever o perfil epidemiológico de gestantes/puérperas soropositivas para o HIV atendidas em uma maternidade pública de referência em gestação de alto risco- no município de Belém-Pará. Métodos: Trata-se de um estudo transversal, observacional de caráter descritivo com abordagem quantitativa. Realizado em uma maternidade de referência em Belém-PA. Resultados: Faixa etária predominante foi de 21 a 25 anos (48,0\%), $80 \%$ se declarou de cor parda, metade é da região metropolitana de Belém e outra do interior do estado, $70 \%$ exercem atividades do lar, $48 \%$ tinham o ensino fundamental incompleto, $70 \%$ vivem em união estável, e $72 \%$ possuem renda familiar de 1 a 3 salários mínimos. Quanto aos hábitos de vida: $12 \%$ fumaram, $18 \%$ consumiram bebidas alcóolicas e apenas $4 \%$ usaram drogas ilícitas/injetável nesta gestação. Conclusão: Conclui-se que o perfil das gestantes puérperas soropositivas se assemelha com os descritos na literatura, por vezes existindo peculiaridades da nossa região. Necessidade de ampliação do diagnóstico de infecção para todas as mulheres jovens, de acordo com as políticas de saúde da mulher.

Palavras-chaves: Transmissão vertical, Grávidas, Vírus HIV.

\begin{abstract}
Objective: To describe the epidemiological profile of HIV-positive pregnant women / postpartum women treated at a public reference maternity hospital in high-risk pregnancy - in the municipality of Belém-Pará. Methods: This is a cross-sectional, observational, descriptive study with a quantitative approach. Held at a referral maternity hospital in Belém-PA. Results: The predominant age group was 21 to 25 years old (48.0\%), $80 \%$ were brown, half were from the metropolitan region of Belém and the other from the interior of the state, $70 \%$ had household activities, $48 \%$ had In incomplete elementary school, $70 \%$ live in a stable union, and $72 \%$ have a family income of 1 to 3 minimum wages. As for life habits: $12 \%$ smoked, $18 \%$ consumed alcohol and only $4 \%$ used illicit / injectable drugs in this pregnancy. Conclusion: It can be concluded that the profile of seropositive pregnant women is similar to those described in the literature, sometimes with peculiarities of our region. Need to expand the diagnosis of infection for all young women, in accordance with women's health policies.
\end{abstract}

Keywords: Vertical transmission, Pregnant, HIV virus.

1 Escola Superior da Amazônia (ESAMAZ), Belém-Pará. *E-mail: adilsonmdfj@hotmail.com

2 Universidade do Estado do Pará (UEPA), Belém-Pará.

3 Universidade Federal do Pará (UFPA), Belém-Pará 


\section{RESUMEN}

Objetivo: Describir el perfil epidemiológico de las mujeres embarazadas / posparto con VIH tratadas en un hospital público de maternidad de referencia en embarazo de alto riesgo, en el municipio de Belém-Pará. Métodos: Este es un estudio transversal, observacional, descriptivo con un enfoque cuantitativo. Celebrada en un hospital de referencia de maternidad en Belém-PA. Resultados: el grupo de edad predominante era de 21 a 25 años (48.0\%), 80\% eran marrones, la mitad eran de la región metropolitana de Belém y el otro del interior del estado, 70\% tenían actividades domésticas, 48\% tenían En una escuela primaria incompleta, el $70 \%$ vive en una unión estable y el $72 \%$ tiene un ingreso familiar de 1 a 3 salarios mínimos. En cuanto a los hábitos de vida: el $12 \%$ fumaba, el $18 \%$ consumía alcohol y solo el $4 \%$ usaba drogas ilícitas / inyectables en este embarazo. Conclusión: se puede concluir que el perfil de las mujeres embarazadas seropositivas es similar al descrito en la literatura, a veces con peculiaridades de nuestra región. Necesidad de ampliar el diagnóstico de infección para todas las mujeres jóvenes, de acuerdo con las políticas de salud de las mujeres.

Palabras-clave: Transmisión vertical, Embarazada, Virus HIV.

\section{INTRODUÇÃO}

Em mais de 30 anos de evolução, a Síndrome da Imunodeficiência Adquirida (AIDS) continua sendo um grave problema de saúde pública, fazendo milhares de vítimas todos os anos e impondo desafios à comunidade científica mundial, como importante fenômeno infeccioso global que representa (NETO et al, 2010).

É relevante destacar que em 1985, para cada 26 casos entre homens havia um caso na mulher. Em 2010 , essa relação foi de 1,7/1, de acordo com Brasil (2011), o que evidencia um processo de feminilização do HIV (Vírus da Imunodeficiência Humana), descrito por Carneiro AJS e Coelho EAC (2010), afirmando que o número de mulheres infectadas aumenta rapidamente entre as heterossexuais casadas, com parceiro único e não-usuárias de drogas, sendo a relação sexual a principal via de transmissão do HIV para essas mulheres.

A partir desse aumento entre as mulheres veio à tona mais um desafio, o controle da transmissão vertical do HIV, visando uma menor incidência de crianças infectadas pelo vírus durante a gestação, parto, puerpério e através do aleitamento materno (BRASIL, 2010). De 1996 até junho de 2009, foram notificados 10.739 casos de AIDS em menores de cinco anos no país, o que representa $2 \%$ do total de casos identificados (BRASIL, 2009).

Embora o Brasil apresente um dos programas mais estruturados de combate ao HIV, o país ainda tem enfrentado grandes desafios na terceira década da epidemia, principalmente no que concerne ao aumento do número de mulheres infectadas, como consequências o aumento da transmissão vertical (TV) (GALVÃO MTG, et al., 2010). Destaca-se, que a TV demonstra um aspecto importante, que é o aumento do número de casos entre crianças devido à infecção dos filhos dessas mulheres, durante o processo de gestação-partoamamentação.

Conforme Brasil (2011), ao longo dos últimos 12 anos observa-se uma estabilização da taxa de incidência no país, mas segundo as regiões a taxa diminuiu na Região Sudeste e aumentou nas demais regiões. Ainda com relação aos números absolutos segundo regiões do país, na Região Norte o maior número de casos de AIDS está no Pará (12.532) correspondendo a $44,4 \%$ do total de casos da região.

O maior número de casos de infecção pelo HIV registrados no país encontra-se na faixa etária de 25 a 49 anos. Do total desses casos $78 \%$ estão no sexo masculino. Para as mulheres, essa proporção corresponde a $71 \%$ (BRASIL, 2009). Este dado demonstra que grande parte das mulheres infectadas pelo HIV se encontra em idade reprodutiva e este contágio pode alterar de muitas formas a experiência da gestação e da maternidade (GONÇALVES TR e PICCININI CA, 2007). Ressalta-se, por sua vez, que entre janeiro de 2000 e junho de 2011, foi notificado no Sistema de Informação de Notificação de Agravos de Notificação (SINAN) um total de 61.789 casos de infecção pelo HIV em gestantes no país, $3.378(5,5 \%)$ na Região Norte dos quais 
1.405 casos $(41,6 \%)$ no Estado do Pará. No ano de 2010, por conseguinte, foram notificados no Brasil 5.666 casos de HIV em gestantes, com taxa de deteç̧ão de 2,0 casos por 1.000 nascidos vivos (BRASIL, 2011).

Nesse contexto, mostra-se relevante realizar um estudo do perfil epidemiológico de gestantes soropositivas para o vírus HIV atendidas em uma maternidade de referência em gestação de alto risco, pois poderá proporcionar uma reflexão crítica acerca da problemática na região, além de possibilitar um debate sobre as políticas públicas que norteiam a temática, e, por conseguinte, tais informações poderão ser utilizadas como ferramentas para os profissionais de saúde na abordagem a essas gestantes.

No que tange aos benefícios o estudo mostra-se relevante para a comunidade científica, haja vista, que permite conhecer o universo dessas gestantes HIV positivo, e, por conseguinte, fornecer reflexões sobre a efetividade das políticas públicas voltadas para a população pesquisada.

O atual estudo teve como objetivo descrever o perfil epidemiológico de gestantes/puérperas soropositivas para o HIV atendidas em uma maternidade pública de referência em gestação de alto risco- no município de Belém-Pará.

\section{MÉTODOS}

Trata-se de um estudo transversal, observacional de caráter descritivo com abordagem quantitativa. Realizado em uma maternidade de referência. Foram convidadas a fazerem parte da pesquisa as gestantes/puérperas soropositivas para o HIV que deram entrada na maternidade no período da coleta de dados ocorrida de agosto a dezembro de2013 e, com o diagnóstico da infecção ou que descobriram ser portadoras do vírus HIV na realização do teste rápido anti-HIV.

As gestantes soropositivas para o HIV que chegaram em franco trabalho de parto ou aquelas em préoperatório imediato de cesárea foram convidadas a participarem do estudo, e a coleta de dados ocorreu nas enfermarias da maternidade, nas quais permanecem en período de pós-parto por aproximadamente 48h, e por sua vez, já se apresentam em sua maioria clinicamente/psicologicamente estáveis. Assim, foram utilizados os seguintes critérios de inclusão: ser gestante e/ou puérpera, independentemente da idade com diagnóstico de infecção pelo HIV e que concordaram em participar do estudo. E como critérios de exclusão: gestantes e/ou puérperas que não apresentaram infecção pelo vírus HIV ou que não aceitaram participar do estudo. Não participariam do estudo gestantes/puérperas sem condições clínicas (pacientes graves em unidade de terapia intensiva, transtorno mental) e/ou que evoluíssem a óbito. No entanto, não ocorreu os casos citados anteriormente.

Foram aplicados testes (Teste qui-quadrado para uma amostra) com intuito de verificar possíveis diferenças significativas entre as frequências observadas nas categorias das variáveis relacionadas à temática.

A interpretação dos testes foi feita de acordo com a convenção cientifica, isto é, o resultado foi chamado de estatisticamente significante se o valor " $p$ " ( $p$-value ou nível descritivo) era menor que 0,05 com nível de significância de $5 \%$.

Todas as gestantes/puérperas da presente pesquisa foram estudadas segundo os preceitos da declaração de Helsinque e do Código de Nuremberg, respeitadas as Normas de Pesquisas envolvendo Seres Humanos (Res. CNS 466/12) do Conselho Nacional de Saúde e foi aprovado por comitê de ética com o número de CAAE: 15317313.5 .0000 .5171$.

\section{RESULTADOS}

$\mathrm{Na}$ tabela 1 se verifica que a faixa etária predominante foi de 21 a 25 anos, representando $48,0 \%, 26$ a 30 anos 22,0\%, até 20 anos 16,0\%, seguido de 31 a $3510,0 \%$ e de 36 a 40 anos 4,0\%. Em relação às medidas estatísticas das idades das gestantes/puérperas, se verificou que a idade variou de 17 a 38 anos, com média de 24,9 anos e desvio-padrão de 4,8. 
Tabela 1- Distribuição das gestantes/puérperas soropositivas para o HIV atendidas na FSCMPA, de acordo com as características socioeconômicas e demográficas, no período de agosto a dezembro de 2013.

\begin{tabular}{|c|c|c|}
\hline Variável & $\mathbf{n}$ & $\%$ \\
\hline \multicolumn{3}{|l|}{ Faixa etária (em anos) } \\
\hline Até 20 anos & 8 & 16,0 \\
\hline 21 a 25 & 24 & 48,0 \\
\hline 26 a 30 & 11 & 22,0 \\
\hline 31 a 35 & 5 & 10,0 \\
\hline 36 a 40 & 2 & 4,0 \\
\hline Total & 50 & 100,0 \\
\hline \multicolumn{3}{|l|}{ Cor } \\
\hline Branca & 7 & 14,0 \\
\hline Negra & 3 & 6,0 \\
\hline Parda & 40 & 80,0 \\
\hline Total & 50 & 100,0 \\
\hline \multicolumn{3}{|l|}{ Procedência } \\
\hline Interior do Pará & 25 & 50,0 \\
\hline RMB & 25 & 50,0 \\
\hline Total & 50 & 100,0 \\
\hline \multicolumn{3}{|l|}{ Ocupação } \\
\hline Do lar & 35 & 70,0 \\
\hline Agricultora & 2 & 4,0 \\
\hline Cozinheira & 2 & 4,0 \\
\hline Estudante & 2 & 4,0 \\
\hline Lavradora & 2 & 4,0 \\
\hline Vendedora & 2 & 4,0 \\
\hline Costureira & 1 & 2,0 \\
\hline Doméstica & 1 & 2,0 \\
\hline Merendeira & 1 & 2,0 \\
\hline Pescaria & 1 & 2,0 \\
\hline Recepcionista & 1 & 2,0 \\
\hline Total & 50 & 100,0 \\
\hline \multicolumn{3}{|l|}{ Escolaridade } \\
\hline Ens. Fund. Incompleto & 24 & 48,0 \\
\hline Ens. Médio Incompleto & 11 & 22,0 \\
\hline Ens. Médio Completo & 15 & 30,0 \\
\hline Total & 50 & 100,0 \\
\hline \multicolumn{3}{|l|}{ Estado civil } \\
\hline Casada & 1 & 2,0 \\
\hline Solteira & 14 & 28,0 \\
\hline União estável & 35 & 70,0 \\
\hline Total & 50 & 100,0 \\
\hline \multicolumn{3}{|c|}{ Renda familiar (Salário Mínimo) } \\
\hline$<1 \mathrm{SM}$ & 14 & 28,0 \\
\hline 1 a 3 SM & 36 & 72,0 \\
\hline Total & 50 & 100,0 \\
\hline
\end{tabular}

Fonte: Dados da pesquisa, 2013. RMB = Ananindeua, Belém, Marituba, Benevides, Santa Isabel do Pará, Santa Bárbara do Pará e Castanhal.

A cor parda foi predominante em $80,0 \%$, seguida da cor branca com $14 \%$ e se consideraram de cor negra $6 \%$ das participantes. Quanto à procedência metade é da região metropolitana de Belém e a outra é procedente do interior do Pará. A grande maioria delas no que concerne à ocupação se considerou do lar $70,0 \%$, e as demais $(30,0 \%)$ desenvolvem outras atividades laborais. Em relação ao grau de instrução quase metade tem apenas o ensino fundamental incompleto $48,0 \%$, seguidas pelas que possuíam o ensino médio completo $30,0 \%$, com ensino médio incompleto $22,0 \%$. Investigando a situação conjugal, constatou-se que união 
estável predominou com $70,0 \%$, enquanto $28,0 \%$ são solteiras e $2,0 \%$ são casadas. No que tange a renda familiar mensal, a predominante foi de 1 a 3 salários mínimos com $72,0 \%$, seguida de $<$ de $1 \mathrm{SM}$, correspondendo a $28,0 \%$.

Na Tabela 2 são apresentadas algumas variáveis referentes aos hábitos de vida das gestantes/puérperas. Verifica-se que 36,0\% delas já utilizaram cigarro e 12,0\% fumaram nesta gestação. Em relação ao consumo de bebida alcoólica se verifica que $62,0 \%$ delas já consumiram ou consomem, mas apenas 18,0\% consumiram durante a gestação. Destas $8,0 \%$ declararam já ter usado droga ilícita, mas apenas 4,0\% afirmaram ter usado nesta gestação. Verifica-se também que houve diferenças significativas na maioria das variáveis $(p<0,05)$, com exceção da variável "Consumo de bebida alcóolica" $(p>0,05)$.

Tabela 2 - Distribuição das gestantes/puérperas soropositivas para o HIV atendidas na FSCMPA, de acordo com os hábitos de vida, no período de agosto a dezembro de 2013.

\begin{tabular}{|c|c|c|c|}
\hline Variável & $\mathbf{n}$ & $\%$ & $\mathbf{p}$ \\
\hline \multicolumn{4}{|c|}{ Uso de cigarro } \\
\hline Não & 32 & 64,0 & 0,0460 \\
\hline Sim & 18 & 36,0 & \\
\hline Total & 50 & 100,0 & \\
\hline \multicolumn{4}{|c|}{ Fumo na gestação } \\
\hline Não & 44 & 88,0 & 0,0001 \\
\hline Sim & 6 & 12,0 & \\
\hline Total & 50 & 100,0 & \\
\hline \multicolumn{4}{|c|}{ Consumo de bebida alcóolica } \\
\hline Não & 19 & 38,0 & 0,1198 \\
\hline Sim & 31 & 62,0 & \\
\hline Total & 50 & 100,0 & \\
\hline \multicolumn{4}{|c|}{ Consumo de bebida alcoólica } \\
\hline \multicolumn{4}{|c|}{ nesta gestação } \\
\hline Não & 41 & 82,0 & 0,0001 \\
\hline Sim & 9 & 18,0 & \\
\hline Total & 50 & 100,0 & \\
\hline \multicolumn{4}{|c|}{ Uso de droga ilícita/injetável } \\
\hline Não & 46 & 92,0 & 0,0001 \\
\hline Sim & 4 & 8,0 & \\
\hline Total & 50 & 100,0 & \\
\hline \multicolumn{4}{|c|}{ Uso de droga ilícita/injetável } \\
\hline \multicolumn{4}{|c|}{ na gestação } \\
\hline Não & 48 & 96,0 & 0,0001 \\
\hline Sim & 2 & 4,0 & \\
\hline Total & 50 & 100,0 & \\
\hline
\end{tabular}

Fonte: Protocolo de pesquisa/agosto a dezembro de 2013. 
A Tabela 3 apresenta as características gineco-obstétricas, onde se verifica que $28,0 \%$ delas já tiveram algum aborto, destas $78,6 \%$ tiveram um aborto e $21,4 \%$ tiveram dois abortos. Pode-se verificar também que $82,0 \%$ já utilizaram algum método contraceptivo, e o mais utilizado foi o preservativo com $31,7 \%$, seguido por oral e preservativo juntos com $17,1 \%$ e apenas oral com 14,6\%. Verifica-se também que houve diferenças significativas em todas as variáveis $(p<0,05)$, com exceção de "Número de abortos" onde não foi possível aplicação de teste devido ao baixo tamanho amostral nesta variável (Tabela 4).

Tabela 3 - Distribuição das gestantes/puérperas soropositivas para o HIV atendidas na FSCMPA, de acordo com as características Gineco-obstétricas, no período de agosto a dezembro de 2013.

\begin{tabular}{|c|c|c|c|}
\hline Variável & $\mathbf{n}$ & $\%$ & $\mathbf{p}$ \\
\hline \multicolumn{4}{|l|}{ Aborto } \\
\hline Não & 36 & 72,0 & \multirow{2}{*}{0,003} \\
\hline Sim & 14 & 28,0 & \\
\hline Total & 50 & 100,0 & \\
\hline \multicolumn{4}{|l|}{ Número de abortos } \\
\hline Um & 11 & 78,6 & \multirow[b]{2}{*}{-} \\
\hline Dois & 3 & 21,4 & \\
\hline Total & 14 & 100,0 & \\
\hline \multicolumn{4}{|l|}{ Uso de método Contraceptivo } \\
\hline Não & 9 & 18,0 & \multirow{2}{*}{0,0001} \\
\hline Sim & 41 & 82,0 & \\
\hline Total & 50 & 100,0 & \\
\hline \multicolumn{4}{|l|}{ Tipo de contraceptivo } \\
\hline Preservativo & 14 & 34,1 & \multirow{7}{*}{0,0047} \\
\hline Oral, preservativo & 7 & 17,1 & \\
\hline Oral & 6 & 14,6 & \\
\hline Injetável & 5 & 12,2 & \\
\hline Injetável, preservativo & 5 & 12,2 & \\
\hline Oral, injetável, preservativo & 3 & 7,3 & \\
\hline Injetável, oral & 1 & 2,4 & \\
\hline Total & 41 & 100,0 & \\
\hline
\end{tabular}

Fonte: Protocolo de pesquisa/agosto a dezembro de 2013.

Tabela 4 - Distribuição das gestantes/puérperas soropositivas para o HIV atendidas na FSCMPA, de acordo com as características da gestação atual, no período de agosto a dezembro de 2013.

\begin{tabular}{|c|c|c|c|}
\hline Variável & $\mathbf{n}$ & $\%$ & $\mathrm{p}$ \\
\hline \multicolumn{4}{|l|}{ Teste anti-HIV nesta gestação } \\
\hline Não & 18 & 36 & 0,066 \\
\hline Sim & 32 & 64 & \\
\hline Total & 50 & 100 & \\
\hline \multicolumn{4}{|l|}{ Período de realizacão do teste HIV } \\
\hline 10 trimestre & 13 & 40,6 & \\
\hline 20 trimestre & 13 & 40,6 & 0,2163 \\
\hline 30 trimestre & 6 & 18,8 & \\
\hline $\begin{array}{ll}\text { Total } \\
\end{array}$ & 32 & 100 & \\
\hline \multicolumn{4}{|l|}{ Diagnóstico de infeccão pelo HIV } \\
\hline Antes do pré-natal & 14 & 28 & \\
\hline Na maternidade & 9 & 18 & 0,0001 \\
\hline Após o parto & 2 & 4 & \\
\hline Durante o pré-natal & 25 & 50 & \\
\hline Total & 50 & 100 & \\
\hline \multicolumn{4}{|l|}{ Status sorológico do parceiro atual } \\
\hline 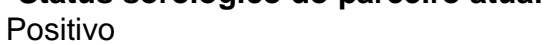 & 15 & 30 & 1,000 \\
\hline
\end{tabular}




\section{DISCUSSÃO}

A partir do aumento dos casos de mulheres infectadas pelo HIV, destaca-se a gestação na presença de uma infecção pelo HIV, pela probabilidade da transmissão vertical. Neste contexto se realizou a presente pesquisa na maior maternidade pública do Norte do Brasil, referência em gestação de alto risco, buscandose conhecer o perfil epidemiológico de gestantes/puérperas soropositivas para o HIV.

O perfil sócio demográfico referente à faixa etária mostrou maior frequência de grávidas infectadas pelo HIV na faixa de 21 a 25 anos 48,0\%, com média de 24,9 anos. Assim como os encontrados por Menezes LSH (2012), entre as grávidas de Belém na FSCMPA, onde houve predomínio da faixa etária entre 18 e 23 anos (42,1\%) com média de 25 anos. No estudo de Lana FCL e Lima AS (2010) no município de Belo Horizonte destacou-se a maior ocorrência de gestações em mulheres adultas jovens na faixa de 25 a 29 anos (média e mediana de 25 anos). Os dados também concordam com os de Santa Catarina, onde a faixa etária predominante das notificações de HIV em gestante é a de 20 a 29 anos 57,0\% (SANTA CATARINA, 2010), bem como de Vieira ACBC, et al. (2011) que encontrou média de 24,3 anos.

No que tange a variável cor/raça, a parda foi predominante em $80,0 \%$, seguida da cor branca com $14 \%$ e se consideraram de cor negra $6 \%$ das participantes. Lana FCL e Lima AS (2010) destacam em sua casuística em Belo Horizonte 57,8\% de gestante de cor não branca, enquanto Vieira ACBC, et al. (2011) encontrou no Espírito Santo para o total de 101 mulheres com dados sobre raça/cor 33,7\% eram pardas, 38,6\% negras e $27,7 \%$ brancas. Nesta pesquisa, metade das gestantes é da região metropolitana de Belém e a outra é procedente do Interior do Pará. A partir dos dados citados acima, verifica-se que, mesmo com a grande concentração de casos na região metropolitana de Belém, é evidente a interiorização da epidemia no Estado, corroborando com Pinto ACS, et al. (2007), que afirma que apesar dos casos se concentrarem nas áreas urbanas e regiões metropolitanas, observa-se um processo de interiorização da infecção no País, para municípios de médio e pequeno porte. Fato este, que para Menezes LSH (2012), demonstra que a presença destas gestantes na capital possivelmente decorre da fragilidade dos municípios em atender às reais necessidades de saúde da comunidade. Para Grangeiro A, et al. (2010), esta situação é uma síntese que a interiorização da epidemia ainda se confronta com a centralização das unidades prestadoras de serviços em AIDS/HIV nos grandes centros urbanos, dificultando o acesso das grávidas residentes em municípios de menor porte.

A análise do grau de instrução das gestantes/puérperas soropositivas para o HIV demonstra que aproximadamente metade (48\%) tem apenas o ensino fundamental incompleto, 30,0\% com ensino médio completo e $22 \%$ com ensino médio incompleto, estas características vão ao encontro de estudos nacionais que identificaram o perfil sócio demográfico dessas gestantes. Figueiró-Filho EA, et al. (2005) em seu estudo prospectivo com 71 gestantes encontrou em relação à escolaridade, o nível fundamental predominante (77\%), seguido do nível médio (19\%), no município de Itajaí (Santa Catarina) houve predomínio também do ensino fundamental $(59,4 \%)$ (KNOLL RK, 2008).

Quando analisado a ocupação, as ocupações de costureira, doméstica, merendeira, pescadora e recepcionista, representaram em igual proporção $12,0 \% \%$. Tal resultado é semelhante ao descrito por Landroni MAS (2004) em seu estudo em São Paulo com 586 mulheres infectadas acompanhadas no serviço de atendimento a pacientes com HIV/AIDS entre 1997 a 2001, onde a ocupação como dona de casa eram as características da maioria das mulheres estudadas. Romanelli RMC, et al. (2006), verificou em seus achados que $61,1 \%$ delas se definiram como trabalhadoras do lar.

De acordo com Santos NJS, et al. (2009) esses dados podem estar relacionados tanto a uma condição de saúde comprometida, quanto aos obstáculos que essas mulheres com HIV enfrentam para entrar e manterse no mercado de trabalho com esse diagnóstico. Além disso, pode-se relacionar que o baixo grau de escolaridade é um fator importante de interferência na obtenção de uma profissão que exija uma maior qualificação, pois o mercado de trabalho requer capacitação.

No grupo de grávidas da pesquisa verificou-se que $70 \%$ vivem em união estável, enquanto $28,0 \%$ são solteiras e 2,0\% são casadas. Tais dados concordam com o estudo realizado na cidade de Blumenau por 
Cobacho KF (2011) que demonstrou que a maioria das entrevistadas mantém uma relação de união estável $53,70 \%, 27,80 \%$ são solteiras, $14,81 \%$ são casadas e 3,70\% são divorciadas. Enquanto Ramos JIM, et al. (2013) evidenciou em 2010, 79\% das gestantes se declararam solteiras, enquanto em $2011,43 \%$ declararam ter uma união estável. Dessa forma, observa-se um aumento no número de gestantes com HIV que possuem uma união estável.

Assim, as evidências nos revelam que a infecção pelo HIV está atingindo cada vez mais mulheres monogâmicas, inclusive as casadas, conforme dados observados entre 1.380 gravidas infectadas pelo HIV (MIRANDA AE, et al. 2009), o que pode mostrar que a mulher brasileira mantém o interesse em relações afetivas que representem estabilidade emocional e familiar, mas que não estão protegidas contra o HIV.

Em relação à renda familiar mensal $72,0 \%$ se enquadraram na categoria de 1 a 3 salários mínimos, e $28,0 \%$ de < de 1 SM (Salário Mínimo). Enquanto Amorim MES (2010), em seu estudo com gestantes soropositivas para HIV/AIDS, atendidas em um serviço público de referência em João Pessoa-PB evidenciaram $74 \%$ delas não possuíam qualquer tipo de renda financeira. A partir do exposto cabe destacar que a renda familiar dessas gestantes, embora esteja enquadrada no intervalo de 1 a 3 salários mínimos para melhor análise, a mesma foi relatada pela maioria como de apenas 1 salário mínimo.

Os achados acima vão ao encontro as análises das tendências da epidemia e seus rumos realizada por Pinto ACS, et al. (2007) que apontam que há relativa tendência de expansão do número de casos entre as populações com baixo nível de renda e escolaridade, atingindo camadas sociais sem nenhuma ou quase nenhuma proteção social, tendência que é denominada por alguns autores como a "pauperização" da epidemia brasileira.

Neste contexto, referente aos hábitos de vida das gestantes/puérperas soropositivas, verifica-se que $36,0 \%$ delas já utilizaram cigarro e 12,0\% fumaram nesta gestação. Quanto ao consumo de bebida alcoólica $62,0 \%$ delas já consumiram ou consomem, mas apenas $18,0 \%$ consumiram durante a gestação. Tais dados apresentam proporções que pode ser comparada aos achados de Lana FCF e Lima AS (2010), onde uso de álcool durante a gestação foi reportado por $16,8 \%$ das gestantes e de tabaco por $23,5 \%$.

Quanto ao uso de drogas ilícitas/injetáveis 8,0\% declararam já ter usado droga ilícita, mas apenas 4,0\% afirmaram ter usado nesta gestação, embora seja um percentual baixo este deve ser destacado, pois se mostrou superior ao achado por Knoll RK (2008) em Santa Catarina, onde 1,9\% das gestantes declararam serem usuárias de drogas injetáveis.

Quanto à história gineco-obstétrica das participantes a média da menarca foi de 13,2 anos. A idade em que tiveram a primeira relação sexual teve média de 15,4 anos. Tal dado converge com a afirmação de Brasil (2006), que destaca que uma das possíveis explicações para a vulnerabilidade desses jovens frente à infecção do HIV/AIDS é o início precoce da vida sexual, somado ao aumento da ocorrência de infecções sexualmente transmissíveis (IST) e à intensificação do consumo de drogas. Em relação ao número de gestações a média foi de aproximadamente 3 gestações $(2,8)$.

Cabe destacar que a análise destes dados é relevante à medida que se pode sugerir que as gestantes/puérperas soropositivas para o HIV podem não estar realizando um acompanhamento eficaz no planejamento familiar que as possa sensibilizar quanto aos riscos envolvidos acerca da transmissão vertical, o que se observa pela média de três gestações. Além, disso os dados alertam para a investigação dos outros filhos das gestantes/púerperas que descobrem sua soropositividade para o HIV em uma gestação atual. Vale ressaltar que não se questiona o direito à maternidade dessas mulheres, mas se elas estão tendo o direito à prevenção e ao planejamento de sua vida reprodutiva.

Pode-se verificar também que $82,0 \%$ das participantes já utilizaram algum método contraceptivo, e o mais utilizado foi o preservativo com $31,7 \%$, seguido por oral e preservativo juntos com $17,1 \%$ e apenas oral com $14,6 \%$. Apesar da maioria das participantes do estudo terem relatado o uso de preservativo, salienta-se que no estudo em questão não foi aprofundada a frequência do uso do preservativo, por conseguinte não se pode afirmar a frequência do mesmo, ou seja, se era utilizado de forma esporádica ou frequentemente. 
Este estudo mostrou que referente à idade gestacional das gestantes/puérperas estudadas a média da idade gestacional foi de 37,1 semanas. A Idade gestacional mais frequente foi de 38 semanas $(30,0 \%)$, seguida por 37 semanas $(18,0 \%)$ e 36 semanas $(16,0 \%)$.

Corroborando com Ramos JIM, et al. (2013), onde o período de duração da gestação de maior incidência, em ambos os anos, foi de 37 a 41 semanas, o que totalizou em 2010,65\% e em 2011, 80\%, ou seja, a maioria das gestações foi a termo.

Os dados acima também estão em consonância com os estudos de Brandão T, et al. (2011) que observou um grande número de partos cesáreos, associados aos nascimentos de idade gestacional acima de 37 semanas $(85,1 \%)$.

Conforme preconizado pelo MS o município deve assegurar à gestante a realização da primeira consulta de pré-natal até o $4^{\circ}$ mês de gestação e realizar, no mínimo, seis consultas de acompanhamento pré-natal preferencialmente uma no primeiro trimestre, duas no segundo e três no terceiro trimestre de gestação (BRASIL, 2000).

No presente estudo também foram analisadas as condições de pré-natal dessas grávidas, que por sua vez, pode possibilitar tanto o diagnóstico precoce de infecção por HIV, quanto uma maior possibilidade de redução da transmissão vertical. Os dados mostraram que 92,0\% realizaram pré-natal e 8,0\% não realizaram, taxa de adesão que demonstra uma boa cobertura da atenção pré-natal.

Ao se analisar a realização do teste anti-HIV verificou-se que $64,0 \%$ fizeram teste anti- HIV nesta gestação e os períodos mais frequentes para realização do teste foram $1^{\circ}$ e $2^{\circ}$ trimestre, ambos com $40,6 \%$ e $18,8 \%$ no 3 o trimestre.

Cabe destacar que dentre os $32,0 \%$ de gestantes que não fizeram o teste anti-HIV estão contidas também gestantes que já sabiam do diagnóstico prévio e, portanto não necessitavam realizar outro exame.

Assim, para Souza Junior PRB, et al. (2004) entre as falhas no processo de detecção da infecção pelo HIV durante a gestação, destacam-se, pelo menos, as seguintes: ausência ou início tardio do acompanhamento pré-natal, sem tempo para obter-se o resultado da sorologia para o HIV e a devida intervenção; atendimento pré-natal adequado, porém sem pedido de teste do HIV; atendimento pré-natal adequado, com realização de teste para o HIV, mas sem resultado do exame laboratorial em tempo hábil ou extravio do mesmo.

Em relação ao momento em que souberam do diagnóstico de infecção pelo HIV, 50,0\% afirmaram que souberam durante o pré-natal, $28,0 \%$ tomaram conhecimento antes do pré-natal, $18 \%$ conheceram seu status sorológico no momento da internação (na maternidade) e 4,0\% souberam após o parto.

Corroborando com Knoll RK (2008), onde foi observado que 55\% das mulheres tiveram seu diagnóstico de infecção pelo HIV durante o pré-natal e $4 \%$ teve o diagnóstico durante o parto ou após o parto.

Dados estes que diferem de outros estudos brasileiros que encontraram maior descoberta da positividade para o HIV antes do pré-natal, assim no estudo de Lana FCF e Lima AS (2010) 44,8\% souberam antes da gestação, Torres (2007) em Porto Alegre observou 52,8\%, Menezes (2012) encontrou taxa superior em seus achados com $75,0 \%$.

\section{CONCLUSÃO}

A descrição e análise dos dados das gestantes / puérperas soropositivas para o HIV do estudo possibilitou traçar o perfil destas gestantes que em são maioria são jovens, nos remetendo ao processo de "juvenalização" da epidemia, oriundas da região metropolitana e do interior do estado, apresentando em sua maioria baixa escolaridade, vivendo em uniões estáveis. Percebeu-se que a atenção pré-natal foi o momento que a maioria das gestantes soube seu status de soropositiva para o HIV o que demonstra a necessidade de ampliação do diagnóstico de infecção para todas as mulheres jovens, de acordo com as políticas de saúde da mulher, e captação dos parceiros de mulheres que convivem com o vírus HIV. 


\section{REFERÊNCIAS}

1. AMORIM MES. Adesão à terapia antirretroviral das gestantes soropositivas para o vírus da imunodeficiência humana em serviço público de referência de João Pessoa - Paraíba / Maria Edilânia Silva Amorim. Dissertação de Mestrado - São Paulo; SP, 2010; 185 p.

2. BRASIL. Ministério da Saúde. Portaria N ․ㅡ570, de 1ำ de junho de 2000.

3. BRASIL. Manual de rotinas para assistência de adolescentes vivendo com HIV/Aids. Secretaria de Vigilância em Saúde. Programa Nacional de DST e Aids. Brasília: Ministério da Saúde, 2006.

4. BRASIL. Ministério da Saúde. Agência Nacional de Vigilância Sanitária. Programa Nacional de DST/AIDS. Epidemiologia AIDS. 2009.

5. BRASIL. Ministério da Saúde. Secretaria de Vigilância em Saúde. Departamento de DST/AIDS e Hepatites Virais. Recomendações para Profilaxia da Transmissão Vertical do HIV e Terapia antirretroviral em Gestantes. Brasília: MS; 2010.146p.

6. BRASIL. Ministério da Saúde. Secretaria de Assistência à Saúde. Boletim Epidemiológico- Aids e DST. Brasília: MS; 2011.

7. BRANDAO T, et al. Características epidemiológicas e nutricionais de gestantes vivendo com o HIV. Rev. Bras. Ginecol. Obstet. 2011; (33)8: 188-195.

8. CARNEIRO AJS, COELHO, EAC. Aconselhamento na testagem anti-HIV no ciclo gravídico-puerperal: o olhar da integralidade. Ciênc. saúde coletiva, Rio de Janeiro, 2011.

9. COBACHO KF. O absenteísmo da realização de exames sorológicos de HIV durante a gestação em pacientes atendidas em um Centro Obstétrico da cidade de Blumenau. Trabalho de Conclusão de Curso. Universidade Regional de Blumenau/ Blumenau, 2011.

10. FIGUEIRÓ-FILHO EA, et al. Perfil epidemiológico da infecção pelo HIV-1 em gestantes do estado de Mato Grosso do Sul - Brasil. j. bras. doenças sex. transm. 2005; 17(4): 281-287.

11. GALVÃO MTG, et al. Dilemas e conflitos de ser mãe na vigência do HIV/Aids. Rev Bras Enferm, Brasília. 2010; 63(3): 371-6.

12. GRANGEIRO A, et al. A epidemia de AIDS no Brasil e as desigualdades regionais e de oferta de serviço. Cad. Saúde Pública, Rio de Janeiro. 2010; (26)12: 23355-2367.

13. GONÇALVES TR, PICCININI CA. Aspectos psicológicos da gestação e da maternidade no contexto da infecção pelo HIV/AIDS. Psicol USP. 2007; 18(3): 113-42.

14. KNOLL RK. Atenção à mulher portadora do hiv/aids no ciclo gravídico no município de Itajaí, Santa Catarina, 2000 2005. Universidade do Vale Itajaí. Dissertação de Mestrado (Mestrado em Saúde e Gestão do Trabalho), 2005.

15. LADRONI MAS. Aids e Gravidez: desafio para cuidado nos serviços de saúde(dissertação). São Paulo: Faculdade de Saúde Pública, Universidade de São Paulo, 2004.

16. LANA FCF, LIMA AS. Avaliação da prevenção da transmissão vertical do HIV em Belo Horizonte, MG, Brasil. Rev. bras. enferm. 2010; (63)4: 587-594.

17. MIRANDA AE, et al. Prevalência de sífilis e HIV utilizando testes rápidos em parturientes atendidas nas maternidades públicas de Vitória, Estado do Espírito Santo. Rev. Soc. Bras. Med. Trop. 2009; (42)4: 386-391.

18. NETO et al, Perfil de adultos infectados pelo vírus da imunodeficiência humana (HIV) em ambulatório de referência em doenças sexualmente transmissíveis no norte de Minas Gerais. Rev Med Minas Gerais. 2010; 20(1): $22-29$.

19. PINTO ACS, etal. Compreensão da pandemia da AIDS nos últimos 25 Anos. DST - J bras Doenças Sex Transm. $2007 ; 19(1): 45-50$.

20. RAMOS JIM, et al. Perfil das gestantes hiv/aids admitidas em uma maternidade de alto risco no município de aracaju/se. Cadernos de Graduação - Ciências Biológicas e da Saúde. 2013; (1)17: 27-38.

21. ROMANELLI RMC, et al. Perfil das gestantes infectadas pelo HIV atendidas em pré-natal de alto risco de referência de Belo Horizonte. Rev. Bras. Saude Mater. Infant. 2006; (6)3: 329-334.

22. SANTA CATARINA. Secretaria de Estado da Saúde. Diretoria de Vigilância Epidemiológica. O perfil epidemiológico da AIDS / Secretaria de Estado da Saúde. - Ed. atual. / por Iraci Batista da Silva, Naura Inês Gandin. - Florianópolis: DIOESC, 2010.

23. SANTOS NJS, et al. Contextos de vulnerabilidade para o HIV entre mulheres brasileiras. Cad. Saúde Pública. 2009; (25)2: s321-s333.

24. SOUZA JUNIOR PRB, et al. Infecção pelo HIV durante a gestação: estudo-Sentinela Parturiente, Brasil, 2002. Rev. Saúde Pública. 2004; (38)6: 764-772.

25. VIEIRA ACBC, et al. Prevalência de HIV em gestantes e transmissão vertical segundo perfil socioeconômico, Vitória, ES. Rev. Saúde Pública. 2011; (45)4: 644-651. 